\title{
Comparison of frictional material models with respect to shear band initiation
}

\author{
F. MOLENKAMP (1985). Géotechnique 35, No. 2, 127-143
}

\begin{abstract}
Mr R. Chambon and Mr J. Desrues, Institut de Mécanique de Grenoble

In his Paper the Author develops an interesting discussion about shear band localization for various constitutive models.

Our intention here is on the one hand to underline a theoretical point concerning the assumption of continuous bifurcation (in the sense of Rice \& Rudnicki (1980)), and on the other hand to comment on the results given about the stiffness ratio at the initiation point, in relation to experimental results obtained in a plane strain apparatus.
\end{abstract}

The Paper provided a parametric study for several elasto-plastic constitutive equations, the main feature of which is to involve an elastic unloading curve with only one elasto-plastic branch. This means that for loading the constitutive equation is

$$
\dot{\boldsymbol{\sigma}}=C^{\mathrm{\varepsilon}}
$$

while for unloading

$$
\dot{\sigma}=C^{u} \dot{\varepsilon}
$$

The notation is the Author's, with the addition of indices 1 and $u$ for loading and unloading respectively.

Although we agree with the conclusion, some comments should be made about some assessments encountered in the Paper.

The Author states that the constitutive tensor $C$ contains the same components outside and inside the band, because the stress state is the same at the initiation of the localization. It should be noted that $C$ does not depend only on the stress $\sigma$ and the hardening parameter but also depends on the direction of the stress rate, defined by

$$
\operatorname{dir} \dot{\boldsymbol{\sigma}}=\frac{\dot{\boldsymbol{\sigma}}}{\|\dot{\boldsymbol{\sigma}}\|}
$$

or on dir $\dot{\varepsilon}$ defined in the same way from the strain rate. In the case under consideration, $C$ has the two possible values $C^{1}$ and $C^{4}$. There is no theoretical reason to assume a priori that bifurcation occurs when the material follows a loading path both inside and outside the band.
From an experimental point of view, there is no more reason for such an assumption: for rocks, Rice \& Rudnicki (1980) suggest that unloading outside the band may occur at the bifurcation point. For soils, the experiments conducted by Desrues (1984) show that for plane strain loading paths the past bifurcation direction of strain increment is somewhat different inside and outside the band; for example, it was observed that the principal directions of the strain increment tensors are significantly rotated inside the distorted zones. Discussion should be made in terms of strain rate at the initiation point, but with the present state of experimental techniques it is only possible to suggest that a sudden divergence of the strain rates inside and outside the band cannot be excluded.

From this short discussion, it can be concluded (see Rice \& Rudnicki, 1980; Chambon \& Desrues, 1984) that in the general case it is also necessary to study the possibility of discontinuous bifurcation, i.e. bifurcation modes for which unloading is possible inside or outside the shear band.

Fortunately, for the problem of shear band localization and for all the constitutive equations studied in the Paper, Rice \& Rudnicki (1980) proved theoretically that continuous bifurcation (plastic loading everywhere) occurs before or simultaneously with discontinuous bifurcation, and so the Author's results are still valid. Another demonstration of a more general result can be found in Chambon (1985).

In conclusion, despite the previous remark, overall we agree with the Author, and our opinion is that the shear band localization study could be a good test to select or elaborate a constitutive equation for soils.

As far as the results of the parametric study are concerned, our own results, limited to the MC/MC model, confirm the Author's on the point that, the less dilatant the material is, the higher is the critical stiffness ratio (Desrues, 1984). The consequence is that loose granular materials should show localization sooner in the hardening regime than do the dense materials. Accordingly, experimental data obtained in plane strain tests on dense and loose sand samples, with stereophotogrammetric monitoring of the strain field, 
showed that localization is observed in the hardening regime in both cases but can be detected immediately in the loose material. Fig. 1 gives a representation of the deviatoric part of the incremental strain field (radius of the Mohr circle) during the successive increments of the test. The correspondence with the stress ratio-axial strain curve is given by the numbers 1, 2, 3, .. in Fig. 2. A typical shear band initiation can be observed during the increment $2-3$, approximately at the middle of the pre-peak part of the curve.

This gives experimental confirmation of the Author's result concerning the influence of the dilatancy ratio $\psi$.
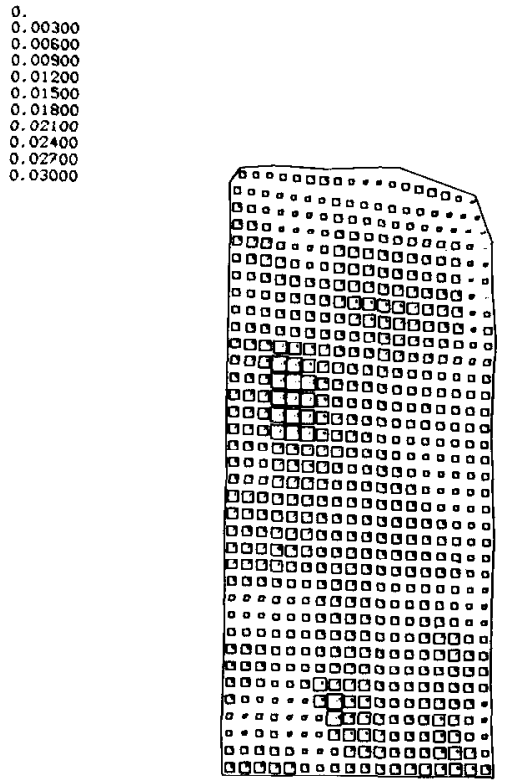
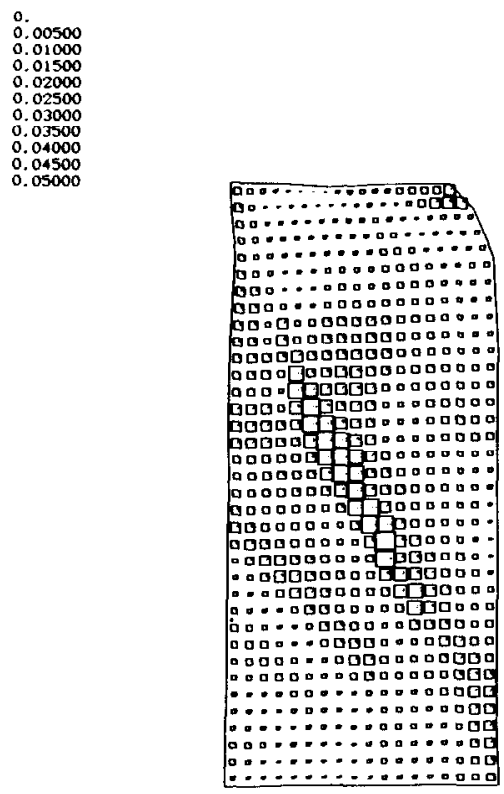

0.00800 0.00800

0.02400

0.03200

0.04800

0.05600

0.07200
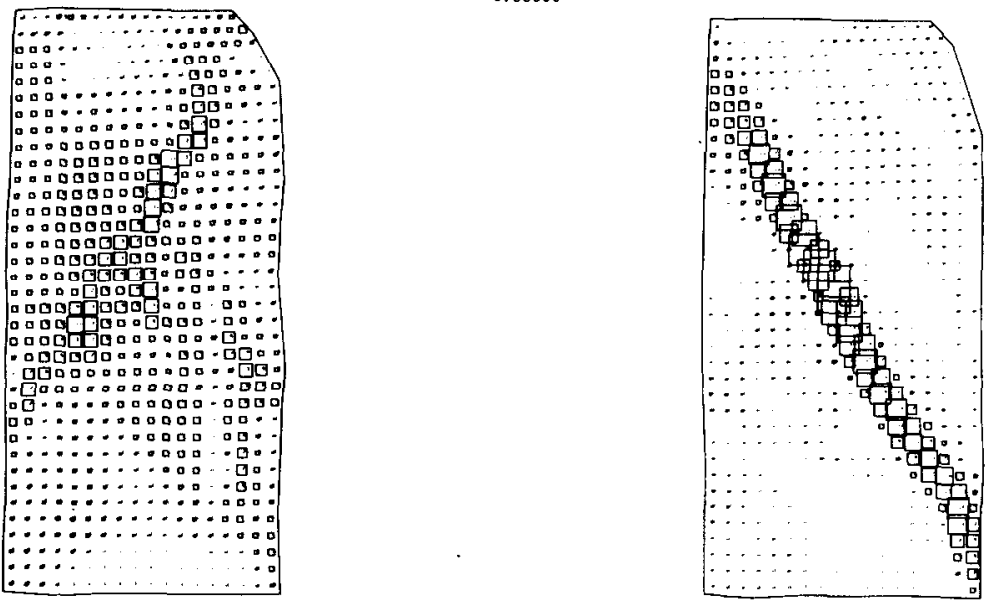

Fig. 1. Incremental distortion maps during test shf03 (loose sand) 


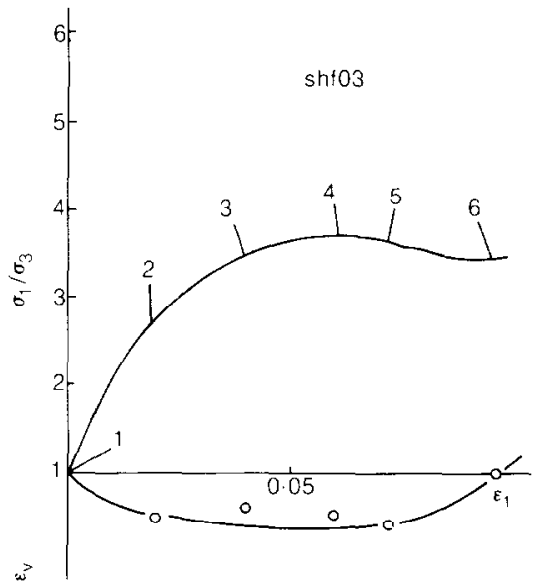

Fig. 2. Test shf03: stress ratio versus axial strain

Our final remark concerns the development of shear bands in a displacement-controlled true triaxial apparatus (rigid platens). Although it is often tacitly assumed that shear bands cannot appear in such an apparatus, but only in apparatuses in which the lowest principal stress is applied via a flexible membrane, this idea is quite false, as has been shown by typical and reproducible results published by Desrues, Lanier \& Stutz (1985).

\section{REFERENCES}

Chambon, R. (1985). Bifurcation par localisation en bande de cisaillement, une approche avec des lois incrémentalement non linéaires. $J . M e ́ c$. Théor Appl., to be published.

Chambon, R. \& Desrues, J. (1984). Quelques remarques sur le problème de la localisation en bande de cisaillement. Mech. Res. Comm. 11, 145-153.

Desrues, J. (1984). La localisation de la déformation dans les materiaux granulaires. Thèse de doctorat d'état, Grenoble.

Desrues, J., Lanier, J. \& Stutz, P. (1985). Localization of the deformation in tests on sand sample. Engng Fract. Mech. 21, No. 4, 909-921.

Rice, J. R. \& Rudnicki, J. W. (1980). A note on some features of the theory of localization of deformation. Int. J. Solids Structs 16, 597-605.

\section{Author's reply}

The Author agrees with the Writers that his assumption of continued loading both inside and outside the shear band was not based on theoretical reasoning. He is grateful to the Writers for providing a reference which gives theoretical support. Also the measurements presented of shear band generation in plane strain tests are very interesting.

The Writers' remark that shear bands are also generated in a displacement-controlled true triaxial apparatus supports the Author's view that at least some existing experimental data must depend partly on the testing machines.

Bifurcation analyses of accurate drained independent stress control tests for stress states near triaxial compression and extension may help to reduce the variation in realistic constitutive models for granular materials. 